\title{
TB RECURRENCE \& TREATMENT OUTCOME AMONG TB PT PUT UNDER DOTS AT CHRISTIAN MEDICAL COLLEGE IN LUDHIANA INDIA
}

Rajinder Kumar Goyal

1. Assistant Professor, Department of Chest \& TB, Christian Medical College \& Hospital, Ludhiana, India

\section{CORRESPONDING AUTHOR}

Dr . Rajinder Kumar Goyal,

Arihant Hospital 238,

Agar Nagar enclave,barewal road,

Ludhiana. Punjab.INDIA

E-mail: rajmonudr@yahoo.co.in

Ph: 00919463258005

ABSTRACT: INTRODUCTION: The DOTS (directly observed treatment short-course) strategy for tuberculosis (TB) control is recommended by the World Health Organization globally. However, there are few studies of long-term TB treatment outcomes from DOTS programs in high-burden settings and particularly settings of high drug resistance. In India adequate studies are yet to be conducted in medical colleges to know about performance of the program, case detection of tuberculosis, cure of the patients registered etc where Revised National Tuberculosis Control Programme (RNTCP) has already been launched. RNTCP was launched in Christian Medical College, Ludhiana, Punjab in February, 2003. OBJECTIVES (i) To evaluate the treatment outcome among cases registered for Directly Observed Treatment Short-course (DOTS) under Designated Microscopy Center (DMC) of Christian Medical College Hospital (ii)To assess various indicators of RNTCP at Christian Medical College Hospital. MATERIALS \& METHODS: A hospital and community based longitudinal study which had both quantitative and qualitative components was carried out in the RNTCP-DMC of Department of Pulmonary Medicine and catchments areas of Ludhiana municipality under DMC of Christian Medical college Hospital, Ludhiana from $1^{\text {st }}$ January,2010 to $31^{\text {st }}$ December 2011. 58 Tuberculosis patients registered and put on DOTS under the DMC of Christian .Medical College were taken as study subjects and they were followed up during their course of treatment to observe their treatment outcome and data analysed using Chi-square test \& proportion. OBSERVATION: A total of 58 patients had been registered in 4 quarters of 2007 for DOTS under DMC of Christian Medical College. There were 29, 11 and 18 patients belonging to Cat-I,Cat-II and Cat-III respectively. Sputum Conversion Rate among new smear positive cases (NSP) was observed to be $82.6 \%$ at the end of Intensive Phase which needs further monitoring in motivating such cases for timely follow-up. Cure rate among new smear positives was calculated to be $78.2 \%$. Treatment success rate was $86.9 \%$ among new smear positive cases in the study. Initial defaulter rate among new smear positives was observed to be $17.4 \%$. Overall default rate was $3.4 \%$ and failure rate was $1.7 \%$ in this study. Death rate among cases was $6.8 \%$. CONCLUSION Low sputum conversion rate at the end of Intensive phase is mainly due to low motivation and low awareness among patients. Low cure rate is due to ignorance of patients who completed their DOTS in time but did not come for final sputum examination. Alcoholism plays the most crucial role behind defaulting consequently leading to deaths recorded in present study.

KEY WORDS: Tuberculosis, RNTCP, DOTS, DOTS Providers(DP). 
INTRODUCTION: World Health Organization Report 2008 on Global Tuberculosis Control states that there were 9.2 million new tuberculosis cases with approximately 1.7 million tuberculosis deaths in 2006. 25\% of global incidences of new TB cases occur in India and India tops the list of 22 High Burden Countries (HBC) in the world followed by China. These High Burden Countries account for more than $80 \%$ of all new TB cases. (1)

The role of medical colleges in TB control as opinion leaders and role models for practicing physicians, in imparting knowledge and skills among medical and paramedical students, shaping their attitude in diagnosing and treating tuberculosis patients cannot be underestimated. Besides benefiting the community directly through the provision of quality services for TB care, their participation can help in sustaining the program by training the future generations of physicians about the principles of DOTS and influencing practices both in the public and private sector. As such, there is a pressing need for all medical colleges to advocate DOTS strategy to provide the best opportunity for cure of the patients.

The involvement of Medical colleges in RNTCP is very important. Their activities primarily include: (i) Training and teaching of RNTCP,(ii) Service delivery of RNTCP through quality assurance net-work group,(iii) Advocacy of RNTCP by sensitization and training through Indian Medical Association,(iv) Operational research by improving DOTS services and managing childhood TB, extra-pulmonary TB and MDR- Tuberculosis.(2)

Use of X-Rays as primary method of diagnosis and use of non-RNTCP regimens for cure are still prevailing in these patients. There is no system of retrieval of irregular and treatment defaulting patients ${ }^{(3)}$.

In India, adequate studies have not been conducted yet in medical colleges to know about performance of the program, case detection of tuberculosis, cure of the patients registered etc at medical colleges where RNTCP has already been launched. Correct information about staffing pattern, their performance, quality of program activities etc is not available.

RNTCP was launched in Christian Medical College, Ludhiana, Punjab in February, 2003. Yet no studies have been conducted on the case detection, registration of patients for DOTS and quality of services provided at Designated Microscopy Centre (DMC) of Christian Medical College in recent past. The current study has been designed and conducted to assess the performance of Revised National Tuberculosis Control Program at Christian Medical College, Ludhiana and to what extent this premier medical college of Punjab has succeeded in case detection, treatment of tuberculosis patients and control of this killer disease in recent past.

MATERIALS \& METHODS: The study was carried out in the RNTCP-DMC of Department of Pulmonary Medicine of Christian Medical College Hospital. The study was carried out from $1^{\text {st }}$ January, 2010 to 31 st December 2011. This was a hospital and community based retrospective longitudinal study which had both quantitative and qualitative components. All of the $58 \mathrm{~TB}$ patients registered under this DMC put on DOTS during 4 quarters of 2010 were taken as study subjects. They were followed up during their course of treatment to assess treatment outcome using both qualitative and quantitative methods. Cat-I and Cat-III patients were followed up thrice and Cat-II patients were followed up 4 times after each follow-up sputum examination during their course of treatment. Primary data from each patient included the demographic profile, sputum smear report, type of tuberculosis, category of treatment regimen and outcome Secondary data were collected from OPD registers, various registers maintained under RNTCP and treatment cards of patients. Different technical and managerial indicators were calculated and analysis was done using appropriate statistical method i.e. Proportions and chi-square test. 
The qualitative data were collected from patients, DPs, MO, STS and STLS through in-depth interviews and focus group discussions.

OBSERVATION: In the year 2010 a total number of 1,31,481 patients had attended different Out Patient Departments of Christian Medical College Hospital. Out of them 724(0.62\%) were chest symptomatic who were referred to RNTCP-laboratory for sputum smear examination. 196 chest symptomatic were found to be positive for Acid-Fast Bacilli.

The number of chest symptomatic referred to RNTCP-laboratory from different OPDs of this medical college has increased many times since implementation of RNTCP in this medical college.724 chest symptomatic were referred to RNTCP-laboratory for sputum examination in 2010 against 133 in 2004. In the year 2010, 724 chest symptomatic were examined. 196 were found sputum positive. 18 out of 196 were put under DOTS at DMC of Christian Medical College while rest 178 New Smear Positive cases were referred for DOTS at other TUs. These 18 cases along with other 40 cases of TB constitute the total study sample of 58 put under DOTS at medical college DMC

$65 \%$ of the patients were males. In present study paediatric cases contribute $1.7 \%$ of total study population. The study revealed that 53 (91.4\%) cases belonged to economically productive age group (15-59 years). Majority of patients belonged to Backward Classes (OBC/SC/ST).

About $35 \%$ of the patients were illiterate or had a low level of education. During an interaction with patients and community members in the study area, it was found that higher the educational status of a person higher the awareness about the mode of the spread and prevention of Tuberculosis. Most of the persons in the community did not know that anti-TB treatment was available free of cost.

$64 \%$ of patients had overcrowding in their household. Average Household size was calculated to be 4.6 and it was $\geq 5$ in $60 \%$ of patients.

$27(46.6 \%)$ patients were daily laborers and lived in poor housing conditions with unsanitary, polluted, urban slum environment. Their low socio-economic status, poor nutrition and lack of awareness about the disease made them vulnerable to Tuberculosis. $40 \%$ of total female cases were house wives. $19 \%$ of patients were dependent sons or daughters which included students, unemployed children etc.

Overall $58.7 \%$ had addiction to any form of tobacco which was observed more among illiterate labor class patients. Smoking which is an important risk factor behind development of Tuberculosis was prevalent among $19 \%$ of patients. $26.3 \%$ male patients and $5 \%$ female cases had history of smoking.

Addiction to alcohol was noted among $22.4 \%$ of the study population and it was found to be a major risk factor behind defaulting and death in this study.

18 NSP cases were administered Cat-I regimen. Out of 13 smear negative pulmonary cases 6 patients were given Cat-I regimen for their serious illness. The rest 7 smear negative cases were given Cat-III regimen. 11 patients who were not seriously ill and suffering from extra-pulmonary disease were put under Cat-III regimen. 47 were new cases while 11 cases were in re-treatment group. There were 29, 11 and 18 TB patients registered for Cat-I, Cat-II and Cat-III treatment regimen respectively.

Sputum conversion rate among NSP cases was $82.6 \%$ at the end of Intensive Phase.

Cure rate among new smear positives was calculated to be $78.2 \%$ (18 out of 23). Cure rate among relapse cases and treatment after default cases were $40 \%$ and $33 \%$ respectively. 
Treatment completion rate among new smear positive cases in this study was $8.6 \%$ (2 out of 23) which is more than RNTCP norm i.e., $\leq 3 \%$. But treatment completion rates among smear-negative and extra-pulmonary cases were $92.3 \%$ and $100 \%$ respectively which shows satisfactory field performance by DPs.

Both cure rate and treatment completion rate contribute towards the treatment success rate among new smear positive cases (86.9\%:20 out of 23). Treatment success rate among relapse cases and 're-treatment after default cases' were $100 \%$ and $33 \%$ respectively.

Initial defaulter rate among new smear positives was observed to be $17.4 \%$. Overall default rate was $3.4 \%$ which is less than the RNTCP norm of 5\%. Various factors as enumerated by DPs behind default were (i) early remission of symptoms which lead to false sense of cure among patients,(ii) early morning hang-over perceived as adverse drug effect of ATT in alcoholic patients,(iii) poor drug compliance due to nausea, vomiting, severe skin rash etc. But adverse drug effects leading to non-adherence to treatment and subsequently to defaulting were not properly and timely reported to corresponding Medical Officer at DMC.

Failure rate in this study was recorded to be $1.7 \%$ which is less than the RNTCP norm of $<4 \%$ of patients put under DOTS. Not a single case of 'failure' was put in re-treatment.

Three of the four dead had discontinued the DOTS and two of them were alcoholic. Death rate in this study was $6.8 \%$ which is beyond RNTCP level of less than $2 \%$. Among the deaths, $75 \%$ belonged to smear positive cases. $50 \%$ of deaths were in Cat-I and other $50 \%$ were in retreatment group. Deaths in Cat-I were due to serious illness of cases, low body weight i.e., $<35 \mathrm{~kg}$ and late diagnosis of the disease.

DISCUSSION: The greatest burden of tuberculosis incidence and mortality in developing countries is in adults aged 15-60 years which includes the most socio-economically productive members of the society such as parents, workers, community leaders etc.Due to their age factor and socio-economic dependence of family they involve themselves in earning and get exposed to other cases in the community.(5)

In an urban area the disease is more related to socio-economic factor, nutritional status, other living conditions like overcrowding, environmental pollution, addiction to smoking etc rather than the caste of the person. TB India 2010, RNTCP Status Report shows that there are $2.88 \%$ and $18.57 \%$ of total population of Ganjam belonging to Scheduled Tribe and Scheduled

CAST RESPECTIVELY: hence the present study reveals the concentration of cases in these sections of community.

The study by Sahu SK et al showed that there is an increase in cure rate and decrease in CDR with an increase in the prevalence of literacy. Median literacy rate of Punjab was reported to be $63.41 \%$ and that of Haryana $60.77 \%$. They recommend to concentrate more on curing the literate TB patients and detecting literate TB cases to have a better figure of Cure rate and Case Detection Rate. ${ }^{(6)}$

In the present study it was also observed that patient with higher literacy had a better concept on disease, importance of treatment adherence \& follow-up sputum examination. The cure rate among 'literates' is more than that of 'illiterates' $(\mathrm{p}<0.001)$.

The addiction to alcohol noted to be $22.4 \%$ in present study was in concordance with the finding ie. $32 \%$ in a study carried out by Jaggarajamma et al. Alcoholism plays the most crucial role in defaulting and consequent deaths in present study. ${ }^{(7)}$ 
The sputum conversion rate among new smear positives registered in the $3^{\text {rd }}$ quarter of 2010 in Punjab and India were reported to be $87 \%$ and $90 \%$ respectively. Low sputum conversion rate in this study (82.6\%) at the end of IP is mainly due to low motivation and low awareness among patients.

Cure rate falls behind the desired RNTCP-norm of $85 \%$ as some of the New Smear Positive patients did not turn up for the end sputum examination.

The cure rate and treatment success rate among NSP cases in Punjab as reported in $1^{\text {st }}$ quarter of 2010 was $82 \%$ and $87 \%$ respectively. The cure rate and treatment success rate among NSP patients registered in $4^{\text {th }}$ quarter of 2009 in India were reported to be $84 \%$ and $86 \%$ respectively. Cure rate and treatment success rate among new smear positives in present study were calculated to be $78.2 \%$ and $86.9 \%$ respectively. However $85.3 \%$ of cured NSP cases had come for 'end of treatment-follow up sputum examination' within 7 days of last dose which is comparably more than the state and country figure of $72 \%$ and $82 \%$ respectively.

Cure rates among relapse cases in Punjab and India were 57.2\% and 66.4\% respectively which were more than that of the study area. Treatment success rate among relapse cases in Punjab and India were $68 \%$ and $73.2 \%$ respectively which were very less than the figure of study area ie. $100 \%$.

Treatment completion has a great role in reducing the chance of emergence of drugresistant bacilli. Many studies have shown that patients who do not complete treatment have isolates which are resistant to the drugs they have taken and these patients infect other people with drug resistant bacilli. ${ }^{(\mathbf{8}, \mathbf{9}}$

Higher rate of initial defaulting (17.4\%) among NSP cases was due to lack of coordination between DMC and DPs as admitted by them in urban area. Defaulting was found in one third of cases registered as 're-treatment after default'. ,

Present study shows that default is due to side effects of drugs(50\%), too many drugs(37.5\%), early relief of symptoms(25\%), non-willingness to come thrice weekly(25\%) for DOTS in IP because of loss of daily earning, visit to relative's house(12.5\%), etc.

This study shows that the risk factors behind death during treatment were male sex(100\%), age $>45$ years(75\%), smear positive(75\%), alcoholism(50\%), previous history of treatment(50\%), body weight at initiation of treatment ie. $<35 \mathrm{~kg}(100 \%)$.

Death rate among re-treatment cases in study population was $18.18 \%$ ( 2 out of 11 ) which was $8.5 \%$ and $7.7 \%$ for the state and country respectively.

CONCLUSION: The case detection rate has increased since implementation of RNTCP in 2003.The case detection would have been more if all of the chest symptomatic from OPD and IPD of department of Paediatrics would have been referred to the RNTCP-DMC for sputum examination.

Irregular treatment, inadequate follow-up action and defaulting are found to be major problems in urban DMC. As alcoholism plays a major role behind drug interruption and deaths, smart and timely reporting on non-adherence to treatment along with proper counseling of these patients will certainly bring them back on DOTS.

Christian Medical College Hospital being a tertiary health care center, the DMC is overburdened with the referral cases from adjacent districts and is not able to give systematic RNTCP services to the specified area as per the program norm which affects the treatment 
outcome. This needs strengthening with additional staff and reorientation of existing functionaries on RNTCP services.

\section{REFERRENCES:}

1. WHO Report 2008, Global Tuberculosis Control; Surveillance, Planning, Financing, World Health Organisation,Genava,2008.

2. Journal of Indian Medical Association, 101: 03, March 2003.

3. Managing the RNTCP in Your Area, A Training Course:Modules1-4,Central TB Division, DGHS, MOHFW, New Delhi, July 1999.

4. RNTCP punjab (1 ${ }^{\text {st }}$ Quarter 2010),State TB Cell,punjab,2010;4-5.

5. Managing the RNTCP in Your Area, A Training Course:Modules5-10,Central TB Division, DGHS, MOHFW, New Delhi, July 1999.

6. Sahu SK , Addresing poverty in TB Control in punjab Journal of community Medicine; July 2010,4:2,3-9.

7. Jaggarajamma $\mathrm{K}$ et al, The Main Reasons for Non-compliance among Patients Treated under RNTCP, Tiruvallur District, South India; Ind Jou of Tuber,July 2007, 54:3, 130-134.

8. RNTCP punjab (1 ${ }^{\text {st }}$ Quarter 2010),State TB Cell,punjab,2010.

9. RNTCP punjab ( $2^{\text {nd }}$ Quarter 2010), State TB Cell,punjab,2010.

Table- I: Socio-demographic profile of study sample $(n=58)$. Characteristics Variables

\begin{tabular}{|l|c|}
\hline 1.Sex & Female \\
Male & $38(65)$ \\
\hline 2.Age Group(years) & $\geq 60$ \\
$0-14$ & $1(2)$ \\
$15-44$ & $44(76)$ \\
$45-59$ & $9(15)$ \\
\hline 3. Caste & $7(12)$ \\
General & $32(55)$ \\
Other Backward Caste & $15(26)$ \\
SC & \\
ST & \\
\hline 4. Literacy & $38(65)$ \\
Literate & \\
\hline
\end{tabular}

\section{Literacy}

Literate

$38(65)$

\begin{tabular}{|l|c|}
\hline $\begin{array}{l}\text { 5.0vercrowding(Persons per living } \\
\text { room>2) }\end{array}$ & Absent \\
Present & $37(64)$ \\
\hline 6.0ccupation & Dependent sons/daughters \\
Laborers & $27(46)$ \\
Government Employee & $7(12)$ \\
Housekeeping & $8(14)$ \\
\hline
\end{tabular}




\begin{tabular}{|c|c|}
\hline Small scale business & $5(9)$ \\
\hline $\begin{array}{l}\text { 7.Socio-economic Status } \\
\text { Low } \\
\text { Medium }\end{array}$ & $\begin{array}{l}\text { High } \\
40(70) \\
16(27)\end{array}$ \\
\hline 8.Knowledge on TB & $\begin{array}{l}\text { Absent } \\
16(28)\end{array}$ \\
\hline $\begin{array}{l}\text { 9.Addiction } \\
\text { Present }\end{array}$ & $\begin{array}{l}\text { Absent } \\
34(59)\end{array}$ \\
\hline
\end{tabular}

Table- II: Distribution of TB patients according to their treatment outcome $(n=58)$.

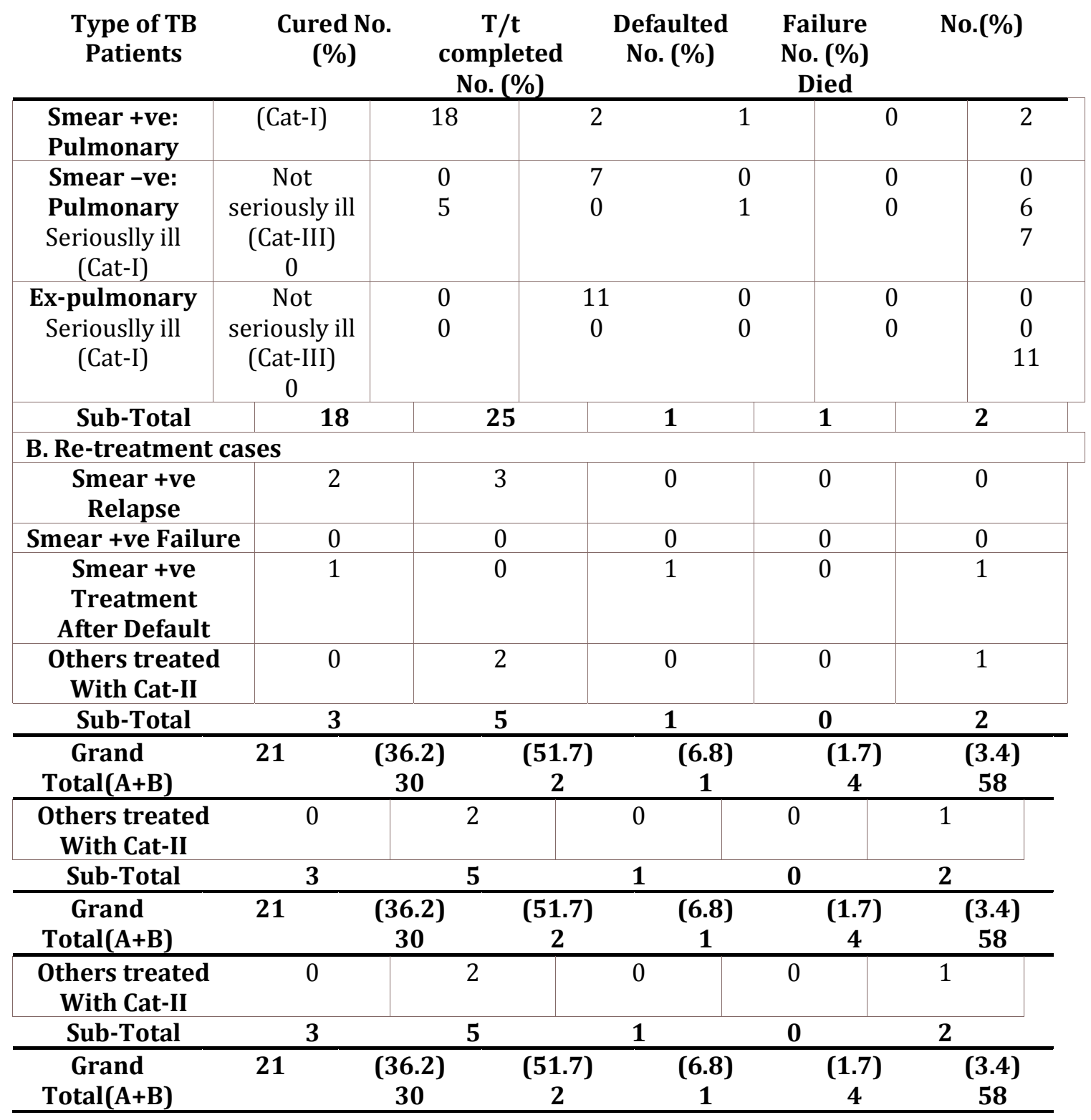

